\title{
The Development of Ultra-high Vacuum Cs-Corrected Scanning Transmission Electron Microscope for Fast Fabrication of Desired Nanostructures
}

Kazuo Furuya, Kazutaka Mitsuishi, Miyoko Tanaka, Masaki. Takeguchi, Yukihito Kondo ${ }^{\dagger}$, Fumio Hosokawa $^{\dagger}$, Kimiharu Okamoto ${ }^{\dagger}$, Takumi Sannomiya ${ }^{\dagger}$, Madoka Hori ${ }^{\dagger}$, Takeshi. Iwama ${ }^{\dagger}$ and Muneyuki Kawazoe ${ }^{\dagger}$

High-voltage electron microscopy station, National Institute for Materials Science, 3-13 Sakura, Tsukuba, Ibaraki, 305-0003, Japan,

${ }^{\dagger} J E O L$ Ltd., 1-2 Musashino 3-chome Akishima Tokyo 196-8558, Japan

Nanofabrication of desired shape and size of structures can be done by the beam assisted process. Electron beam induced deposition (EBID) is an unique technique to produce subnanometer-sized and position-controlled structures. It has been shown that free-standing nano-wires of less than 10 $\mathrm{nm}$ in thickness can be fabricated using EBID [1,2]. The minimum size of fabricated deposits was shown to be about $1.5 \mathrm{~nm}$ in diameter with fine electron beam by ultra-high vacuum TEM (UHVTEM) [3]. For this experiment, it is essential to fabricate in UHV to control precursor gas pressure precisely and to eliminate the contamination in the chamber. Fig. 1 shows typical examples of tungsten nano-dots fabricated by an UHV-TEM. The left image reveals four dots of $2 \mathrm{~nm}$ in diameter with 5 to $15 \mathrm{~s}$ exposure. The right images shows an array of dots formed with a $1 \mathrm{~nm}$ probe and a deposition period of $5 \mathrm{~s}$. The dots are located in the intersecting points of the white lines. They are too small to distinguish them from the amorphous substrate. A high angle annular dark field (HAADF) STEM image (right bottom) shows a clear Z-contrast of tungsten dots. However, the time required to fabricate a dot is relatively long, which is in the range between one and $20 \mathrm{~s}$. Therefore, it is strongly desired to increase the electron beam intensity for fast nanofabrication.

We are now developing an UHV-compatible Cs corrector for the probe forming system of STEM (JEM-2500) [4], not only for a smaller probe for better resolution, but also for higher probe current which will results in faster nanofabrication. Fig. 2 shows a photogarph and schematics of the UHV Cs-corrector with STEM column. The corrector consists of two stages, each one of which has 12 poles and transfer lenses. The 12 poles mainly act as a two sextuple corrector and it can also be excited as two dipoles and two quadrupoles to compensate beam shifts and stigmatisms. The designed performance is as follows: Accelerating voltage is $200 \mathrm{kV}$, Cs can be changed from 0 to $0.7 \mathrm{~mm}$ (uncorrected $\mathrm{Cs}$ is $0.7 \mathrm{~mm}$ ), Cc is $1.6 \mathrm{~mm}$ (after Cs corrected) and UHV specimen vacuum is less than $1.0 \times 10^{-7} \mathrm{~Pa}$. The obtained Ronchigram (in Fig. 3) showed an extension of sweet spot area indicating the successful correction of third order spherical aberration Cs. The expected probe current is quite large (about 10 times larger) as compared with uncorrected probe.

References

[1] M. Shimojo et. al, J. Vac. Sci. Technol. B 22 (2004) 742.

[2] M. Shimojo et. al, Appl. Phys. A 79 (2004) 1869.

[3] M. Tanaka et. al, Surf. Interface Anal., 37 (2005) 261.

[4] K. Mitsuishi et. al, to be published in Micrsco. Microanal. 


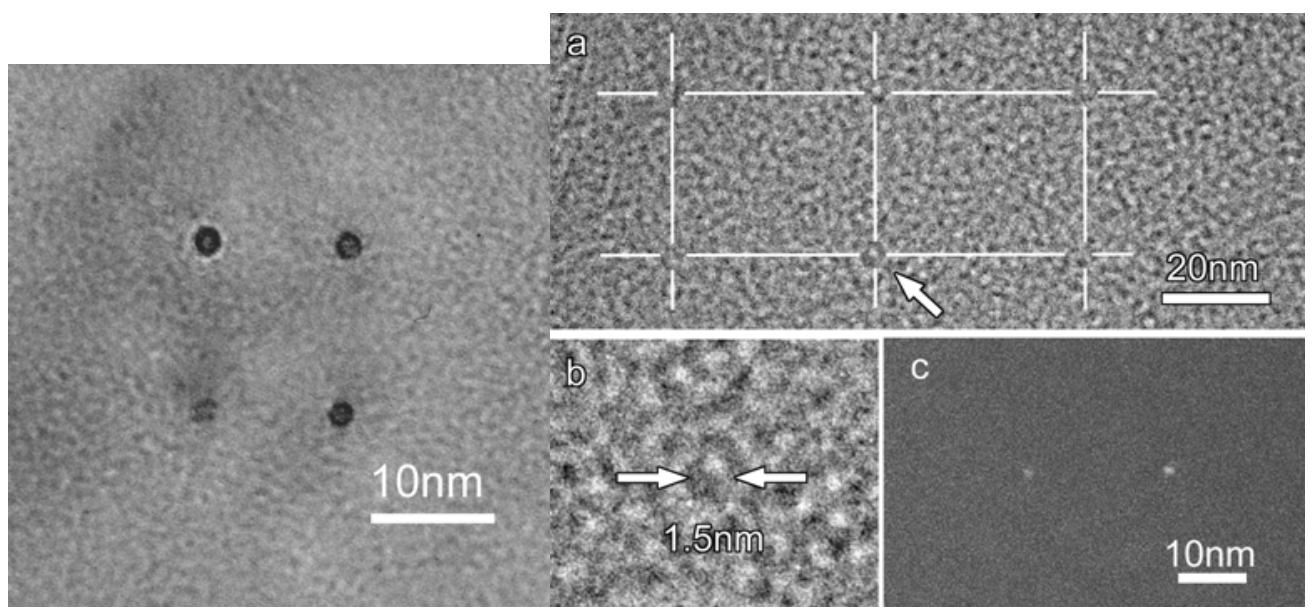

Fig. 1 TEM micrographs of the $\mathrm{W}$ nano-dots with different deposition time. The left image is fabricated for $5-15 \mathrm{~s}$ and the right images are an array of the dots of about $1.5 \mathrm{~nm}$ in diameter.
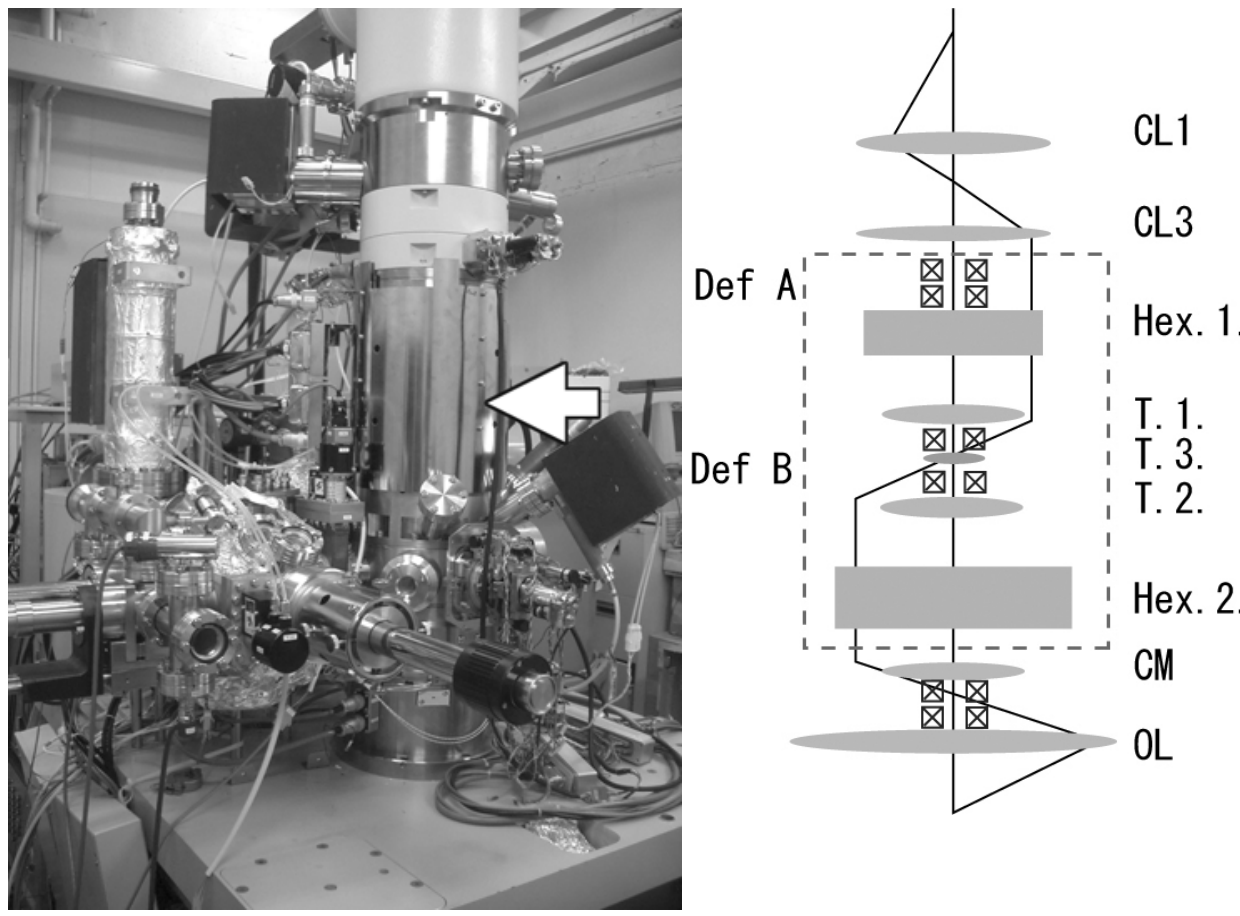

Fig. 2 A photogarph and schematics of the UHV Cs-corrector with STEM column.

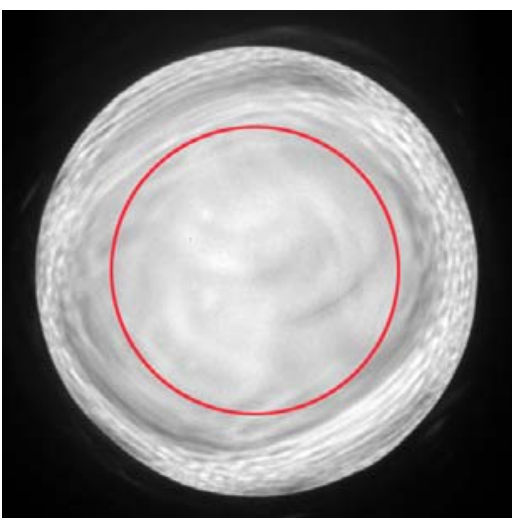

\section{Cs corrected probe and current}

\begin{tabular}{|l|r|r|r|}
\hline Probe size (nm) & 0.2 & 0.5 & 1.0 \\
\hline FWHM & 0.22 & 0.52 & 1.0 \\
\hline D50 & 0.2 & 0.48 & 0.98 \\
\hline D75 & 0.26 & 0.68 & 1.4 \\
\hline D90 & 0.34 & 0.88 & 1.8 \\
\hline D95 & 0.38 & 0.98 & 2.0 \\
\hline Probe Current (nA) & 1.6 & 9.9 & 39.8 \\
\hline
\end{tabular}

Cs $=1 \mu \mathrm{m}, \alpha=40 \mathrm{mrad}, \mathrm{Df}=-1.9 \mathrm{~nm}$

$\mathrm{Cc}=1.1 \mathrm{~mm}, \Delta \mathrm{E}=0.7 \mathrm{eV}$

Fig. 3 The obtained Ronchigram using the Cs-corrector and the estimated electron beam intensities with various Cs corrected probes. The circle is at the $45 \mathrm{mrad}$ position. 